\title{
Alpha2B-Adrenergic Receptor Regulates Neutrophil Recruitment in MSU-Induced Peritoneal Inflammation
}

\author{
Lihua Duan ${ }^{1,2 \dagger}$, Jie Chen ${ }^{1,2 \dagger}$, Michael Razavi ${ }^{2}$, Yingying $\mathrm{Wei}^{2,3}$, Ying Tao ${ }^{3}$, Xiaoquan Rao ${ }^{2,4 *}$ \\ and Jixin Zhong ${ }^{2 *}$ \\ ${ }^{1}$ Department of Rheumatology and Clinical Immunology, Jiangxi Provincial People's Hospital, Nanchang, China, \\ ${ }^{2}$ Cardiovascular Research Institute, School of Medicine, Case Western Reserve University, Cleveland, OH, United States, \\ ${ }^{3}$ School of Medicine, Xiamen University, Xiamen, China, ${ }^{4}$ Oregon Institute of Occupational Health Sciences, Oregon Health \& \\ Science University, Portland, OR, United States
}

OPEN ACCESS

Edited by:

Song Guo Zheng,

Penn State Milton S. Hershey Medical

Center, United States

Reviewed by:

Qing-Sheng Mi, Henry Ford Health System,

United States

Yan Yang,

Rutgers, The State University of

New Jersey, United States

*Correspondence:

Xiaoquan Rao

RaoX@ohsu.edu

Jixin Zhong

Jixin.Zhong@case.edu

tThese authors have contributed equally to this work

Specialty section: This article was submitted to Immunological Tolerance and

Regulation,

a section of the journal

Frontiers in Immunology

Received: 29 December 2018 Accepted: 25 February 2019

Published: 19 March 2019

Citation:

Duan L, Chen J, Razavi M, Wei Y,

Tao Y, Rao X and Zhong J (2019)

Alpha2B-Adrenergic Receptor Regulates Neutrophil Recruitment in

MSU-Induced Peritoneal

Inflammation. Front. Immunol. 10:501.

doi: 10.3389/fimmu.2019.0050
Gout is one of the most common metabolic disorders in human. Previous studies have shown that the disease activity is closely associated with sympathetic nervous system (SNS). $\alpha_{2 B}$-adrenergic receptor $\left(\alpha_{2 B} A R\right)$, a subtype of $\alpha 2$ adrenergic receptor, plays a critical role in many diseases. However, the role of $\alpha_{2 B} A R$ in the pathogenesis of gout remains unclear. Here, we assessed the role of $\alpha_{2 B} A R$ in the monosodium urate (MSU) crystals-induced peritonitis that mimics human gout by using the $\alpha_{2 B} A R$-overexpressing mice $\left(\alpha_{2 B} A R-T g\right)$. We found that the number of recruited neutrophils was significantly increased in the $\alpha_{2 B} A R-T g$ mice after MSU treatment, when compared with wild type mice. In contrast, the number of macrophages was not changed. Importantly, there is no difference in the IL-1 $\beta$ levels and caspase- 1 activity between wild type and $\alpha_{2 B}$ AR-Tg mice in the gout animal model. Notably, the enhanced neutrophil migration in $\alpha_{2 B} A R-T g$ mice was dependent on the $\alpha_{2 B} A R$ overexpression in neutrophils, but not resulted from other tissues or cells with $\alpha_{2 B} A R$ overexpression. In conclusion, our data provide a direct evidence that $\alpha_{2 B} A R$ plays a critical role in neutrophil migration and MSU-induced inflammation.

Keywords: gout, adrenergic receptor, neutrophil, monosodium urate, inflammation

\section{INTRODUCTION}

The cross-talk between the sympathetic nervous system (SNS) and immune system has gained increasing attentions through catecholamines hormone (1-3). Catecholamines exert their physiological effects by binding adrenergic receptors, which are a class of $G$ protein-coupled receptors. There are two main groups of adrenoreceptors, $\alpha$ and $\beta$, with 9 subtypes in total (4). An increased production of endogenous catecholamines epinephrine and norepinephrine can be observed in a status of anxiety, pathogenic challenge, and pain (5). It has been demonstrated recently that SNS play an important role in the induction of stress-induced proinflammatory cytokines, such as IL- $1 \beta$ induction (6). Consistent with this, the baseline epinephrine in healthy humans is inversely correlated with pro-inflammatory cytokine levels and positively with anti-inflammatory cytokine productions (7). In addition, SNS have also been implicated in chronic intestinal inflammation, multiple sclerosis, and rheumatoid arthritis through $\mathrm{T}$ cell immune responses (8-11). 
Accumulating data indicate that SNS plays a critical role in the control of metabolic and cardiovascular homeostasis. Both experimental and clinical studies have shown that the changes of catecholamines in plasma and tissue were associated with the pathogenesis of metabolic and cardiovascular diseases $(12,13)$. Gout is one of the most common human metabolic diseases characterized by sudden pain and swelling in the joints. The abnormal uric acid (UA) metabolism results in monosodium urate (MSU) crystal formation in the joint and periarticular tissues, which activates the resident macrophage to produce IL$1 \beta$, a crucial inflammatory cytokines in the pathogenesis of gout (14, 15). Increasing evidences have demonstrated that $\alpha_{2} \mathrm{AR}$ stimulation promotes cytokine production. It has been shown that the $\alpha_{2} \mathrm{AR}$ was required for norepinephrine-induced DC endocytosis through modulating the PI3K and ERK1/2 signaling pathway in DCs (16). The $\alpha_{2} \mathrm{AR}$ agonist dexmedetomidine can decrease the IL- $1 \beta$-induced IL- 6 production in glioma cells (17). $\alpha_{2}$ ARs include three highly homologous subtypes, $\alpha_{2 \mathrm{~A}} \mathrm{AR}$, $\alpha_{2 B} A R$, and $\alpha_{2 C} A R$. However, the modulation of $\alpha_{2 B} A R$ on the pathogenesis of gout remains elusive.

Here, we addressed the role of $\alpha_{2 B} A R$ in the pathogenesis of MSU-induced inflammation by using the $\alpha_{2 B} A R$ overexpressing transgenic mice $\left(\alpha_{2 B} A R-T g\right)$. We found that the MSU-induced inflammation was exacerbated in $\alpha_{2 B} \mathrm{AR}-\mathrm{Tg}$ mice, characterized by increased neutrophils infiltration. In consistency, neutrophils with $\alpha_{2 B}$ AR over-expression also displayed an enhanced migratory ability in in vitro experiments. In contrast, the number of macrophage and IL-1 $\beta$ levels were not altered. In conclusion, $\alpha_{2 B} \mathrm{AR}$ plays an important role in MSU-induced inflammation by enhancing the migration of neutrophils. Therefore, $\alpha_{2 B} \mathrm{AR}$ may serve as a promising therapeutic target for gout.

\section{MATERIALS AND METHODS}

\section{Animals}

$\mathrm{FVB} / \mathrm{NJ}$ mice (wild-type, $\mathrm{Wt}$ ) and $\alpha_{2 \mathrm{~B}} \mathrm{AR}$ transgenic mice $\left(\alpha_{2 B} \mathrm{AR}-\mathrm{Tg}\right)$ in $\mathrm{FVB} / \mathrm{NJ}$ background were purchased from the Jackson Laboratory. The mice were housed in the specific pathogen-free animal facility at the Case Western Reserve University. All experimental procedures involving mice were approved by the Institutional Animal Care and Use Committees (IACUCs) at the Case Western Reserve University and Xiamen University.

\section{Establishment of Acute Gout Animal Model}

Male mice (6-8 weeks old) were administered an intraperitoneal (IP) injection of $3 \mathrm{mg}$ MSU (Invivogen, US). After $16 \mathrm{~h}$, the peritoneal exudate cells (PECs) and lavage fluids were harvested and subjected to flow cytometry analysis and cytokine assay.
A

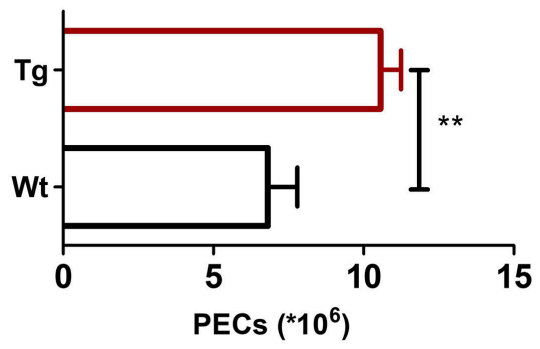

C

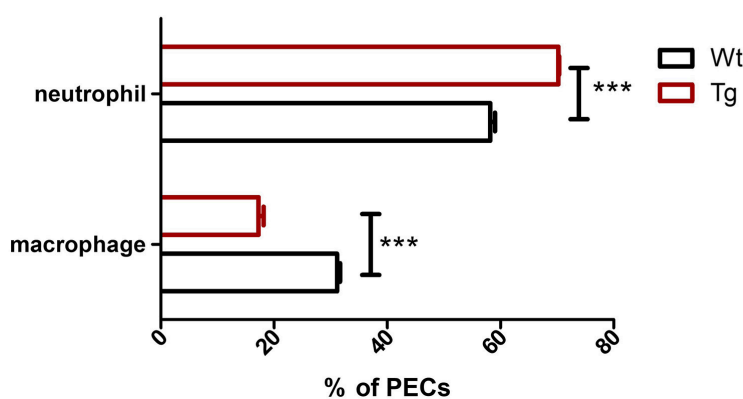

B

D
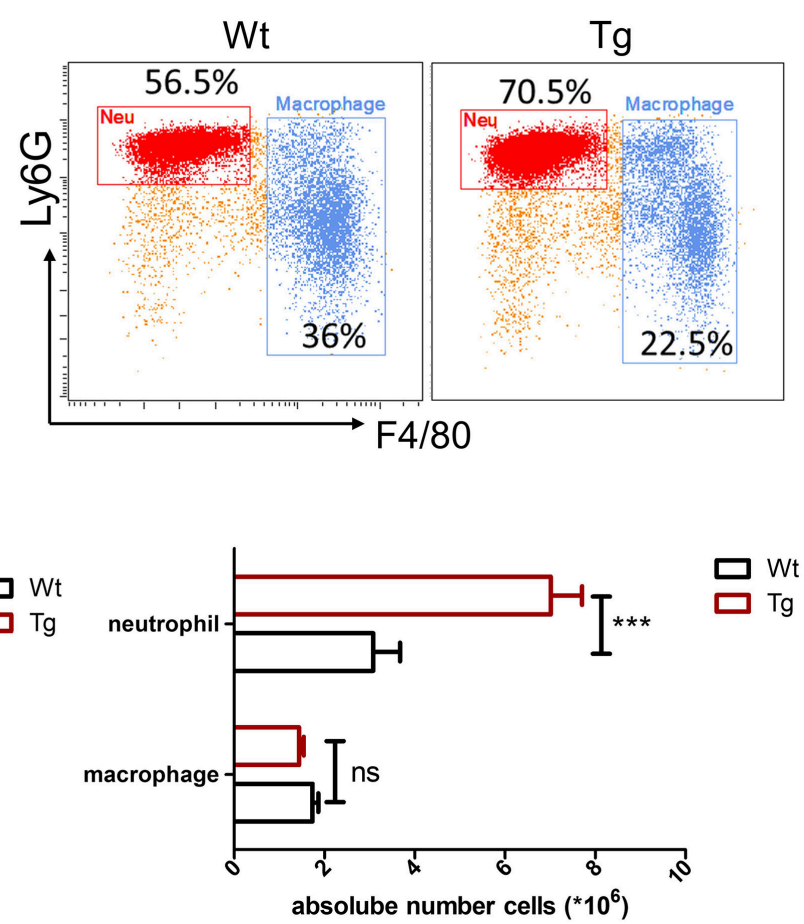

FIGURE 1 | Increased neutrophil recruitment in $\alpha_{2 B} A R-T g$ mice after MSU stimulation. Wt and $\alpha_{2 B} A R-T g$ mice were treated with MSU to induce peritonitis. After $6 \mathrm{~h}$, the total PECs were harvested. (A) Quantitative analysis of total number of PECs. (B) The PECs were stained with anti-CD11b, anti-F4/80, and anti-Ly6G antibodies. CD11 $b^{+}$cells were gated for the analysis of macrophage (CD11b+ Ly6G ${ }^{-}$F4/80 $\left.{ }^{+}\right)$and neutrophil $\left(C D 11 b^{+}\right.$Ly6G $^{+}$F4/80 $\left.{ }^{-}\right)$. Representative images were shown. (C) Statistical bar graph showing the percentages of macrophages and neutrophils in total CD11b+ cells. (D) The absolute numbers of neutrophils and macrophages in PECs. Data are represented as mean $\pm \operatorname{SEM}\left(n=6-8 /\right.$ group). The results shown are from one of three independent experiments. ${ }^{* \star} p<0.01$, ${ }^{\star \star \star} p<0.001$. ns, not significant. 


\section{Flow Cytometry Analysis}

The peritoneal exudate cells (PECs) were incubated with the fluorescent-conjugated monoclonal antibodies in the staining buffer. Antibodies used for flow cytometry are as follows, FITC anti-mouse F4/80, PE anti-mouse CD11b and PE/Cy7 antimouse Ly6G. All antibodies are purchased from Biolegend (San Diego, CA, US). Caspase 1 activity were determined by FAMFLICA Caspase Assay Kits (Immunochemistry Technologies, Bloomington, MN, USA) according to the manufacturer's instructions through flow cytometry analysis.

\section{Mouse Bone Marrow-Derived Macrophage Generation}

Bone marrow-derived macrophages (BMMs) were generated from the bone marrow cells as described previously (18). Briefly, bone marrow cells isolated from the animals were seeded at the concentration of $1 \times 10^{6} / \mathrm{ml}$ cells and cultured in the presence of M-CSF (20 ng/ml) (Peprotech, US) for 7 days. The supernatant was replaced every 2 days. Adherent BMMs were harvested at day 7 for further study.

\section{Quantitative Real Time RT-PCR}

Total RNA was extracted from PECs by TRIzol ${ }^{\circledR}$ Reagent (Invitrogen, Carlsbad, CA) as instructed. Reverse transcription of total RNA was performed using cDNA synthesis kit (ABI, ON, Canada). The primers for $\beta$-actin, IL-1 $\beta$, MIP-2, NLRP3, and $\alpha_{2 B}$ AR gene are listed below. mip-2, forward: TCA ATG CCT GAA GAC CCT G, reverse: CCT TGA GAG TGG CTA TGA CTT C; adra2b, forward: CTG GGC TAT TGG TAC TTC TGG, reverse: AGT TGT ACT CCA ATG CTC GG; $\beta$-actin, forward: ACC TTC TAC AAT GAG CTG CG, reverse: CTG GAT GGC TAC GTA CAT GG; IL-1 $\beta$, forward, ACG GAC CCC AAA AGA TGA AG, reverse: TTC TCC ACA GCC ACA ATG AG; nlrp3, forward: CTC CAA CCA TTC TCT GAC CAG, reverse: ACA GAT TGA AGT AAG GCC GG. The expression of target genes was normalized to $\beta$-actin.

\section{ELISA (Enzyme-Linked Immunosorbent Assay)}

The concentration of IL- $1 \beta$ in the peritoneal lavage fluid or bone marrow macrophage culture supernatant was determined by an ELISA Kit (R\&D, Minneapolis, MN, US) according to the Manufacturer's instruction.

\section{Neutrophil Migration Assay}

Bone marrow neutrophils were isolated from $\mathrm{Wt}$ and $\alpha_{2 \mathrm{~B}} \mathrm{AR}-$ Tg mice using a MojoSortTM Mouse Neutrophil Isolation Kit (Biolegend, US). The isolated $\alpha_{2 B} \mathrm{AR}-\mathrm{Tg}$ and $\mathrm{Wt}$ neutrophils
A

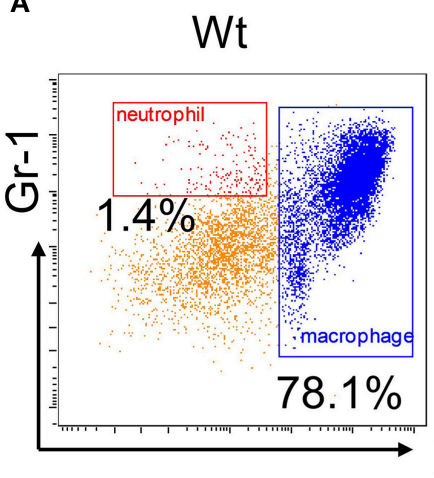

B

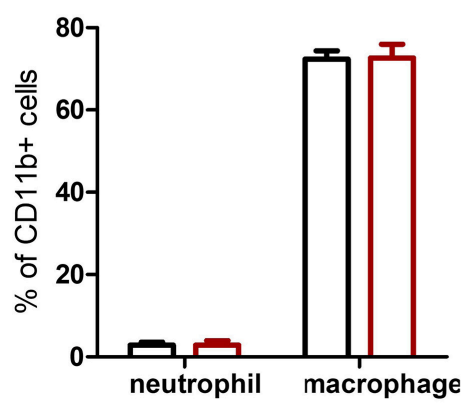

$\mathrm{Tg}$

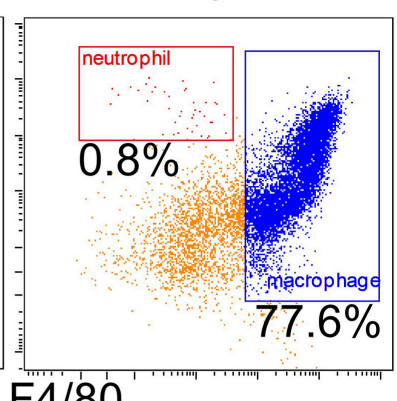

F4/80

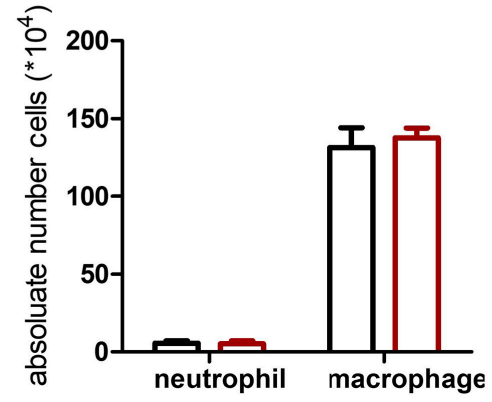

C

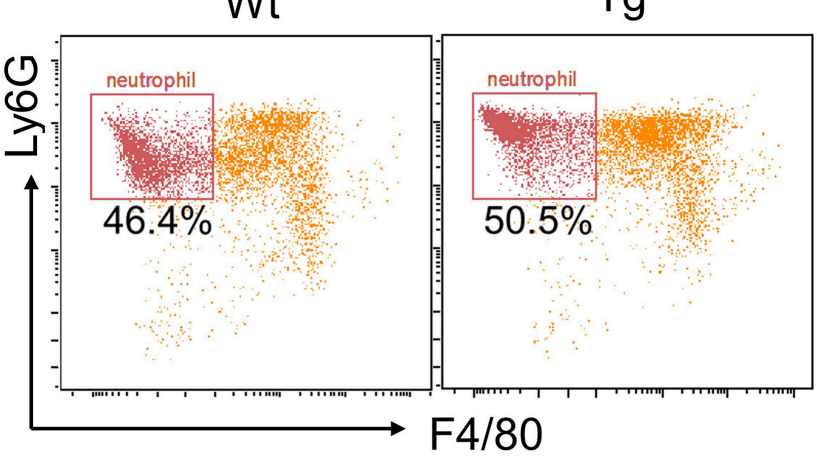

D

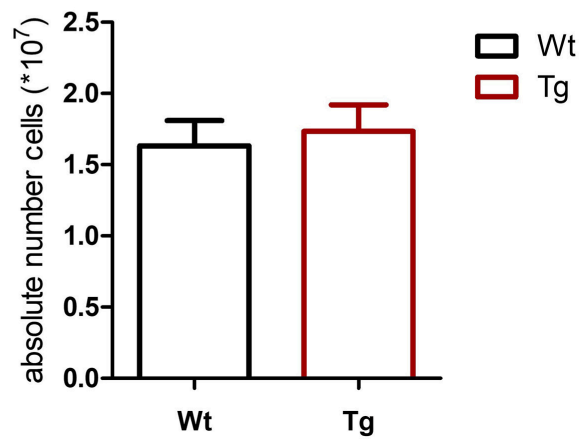

FIGURE $2 \mid \alpha_{2 B} A R$ over-expression does not affect the development of macrophage and neutrophil. The PECs and bone marrow cells were collected from untreated $\alpha_{2 B}$ AR-Tg and Wt mice. (A,B) Percentage of CD11b+F4/80+ macrophages in PECs. The cells were stained with anti-CD11b, anti-F4/80, and anti-Gr1 antibodies. The CD11b+ cells were gated for analysis. The percentage and absolute number of CD11 ${ }^{+} \mathrm{F}_{4 / 80^{+}}$macrophages and CD11 ${ }^{+} \mathrm{Gr}^{+}{ }^{+} \mathrm{F} 4 / 80^{-}$neutrophils in PECs were presented. (C,D) Bone marrow cells were stained with anti-CD11b, anti-F4/80, and anti-Ly6G antibodies. The percentage and absolute number of $\mathrm{CD} 11 \mathrm{~b}^{+} \mathrm{Ly}_{6 \mathrm{G}}{ }^{+} \mathrm{F} 4 / 80^{-}$neutrophils in bone marrow cells was shown. The results shown are from one of three independent experiments $(n=6-8 / \mathrm{group})$. 
were stained with CellTrace ${ }^{\mathrm{TM}}$ Violet (V440) or CellTrace $\mathrm{C}^{\mathrm{TM}}$ CFSE, respectively. Labeled cells were mixed at the at the ratio of 1:1 and then adoptively transferred into MSU-pretreated (3 mg/per mouse) Wt or $\alpha_{2 B} \mathrm{AR}-\mathrm{Tg}$ mice. Splenocytes and PECs were harvested after $6 \mathrm{~h}$. The ratio and numbers of Wt (CFSE) and $\alpha_{2 B}$ AR-Tg (V440) cells in the spleen and peritoneal fluid were determined by flow cytometry. For the in vitro neutrophil migration, the mixed neutrophils in Dulbecco modified Eagle medium with 10\% Fetal Bovine
Serum were placed in the insert of a $6-\mu \mathrm{m}$ Transwell ${ }^{\circledR}$ plate (Thermo Scientific) at the concentration of $1 \times 10^{6}$ cells/mL (100 $\mu \mathrm{l} / \mathrm{well})$. The lower chamber contained 600 ul Dulbecco modified Eagle medium with 10\% Fetal Bovine Serum and MIP-2(200 ng/ml, PeproTech). After incubation for $4 \mathrm{~h}$ in $37^{\circ} \mathrm{C}$, the cells in the insert and bottom wells were collected and the ratio of Wt (CFSE) vs. $\alpha_{2 B} \mathrm{AR}-\mathrm{Tg}$ (V440) cells were detected by fluorescence microscope and flow cytometry.
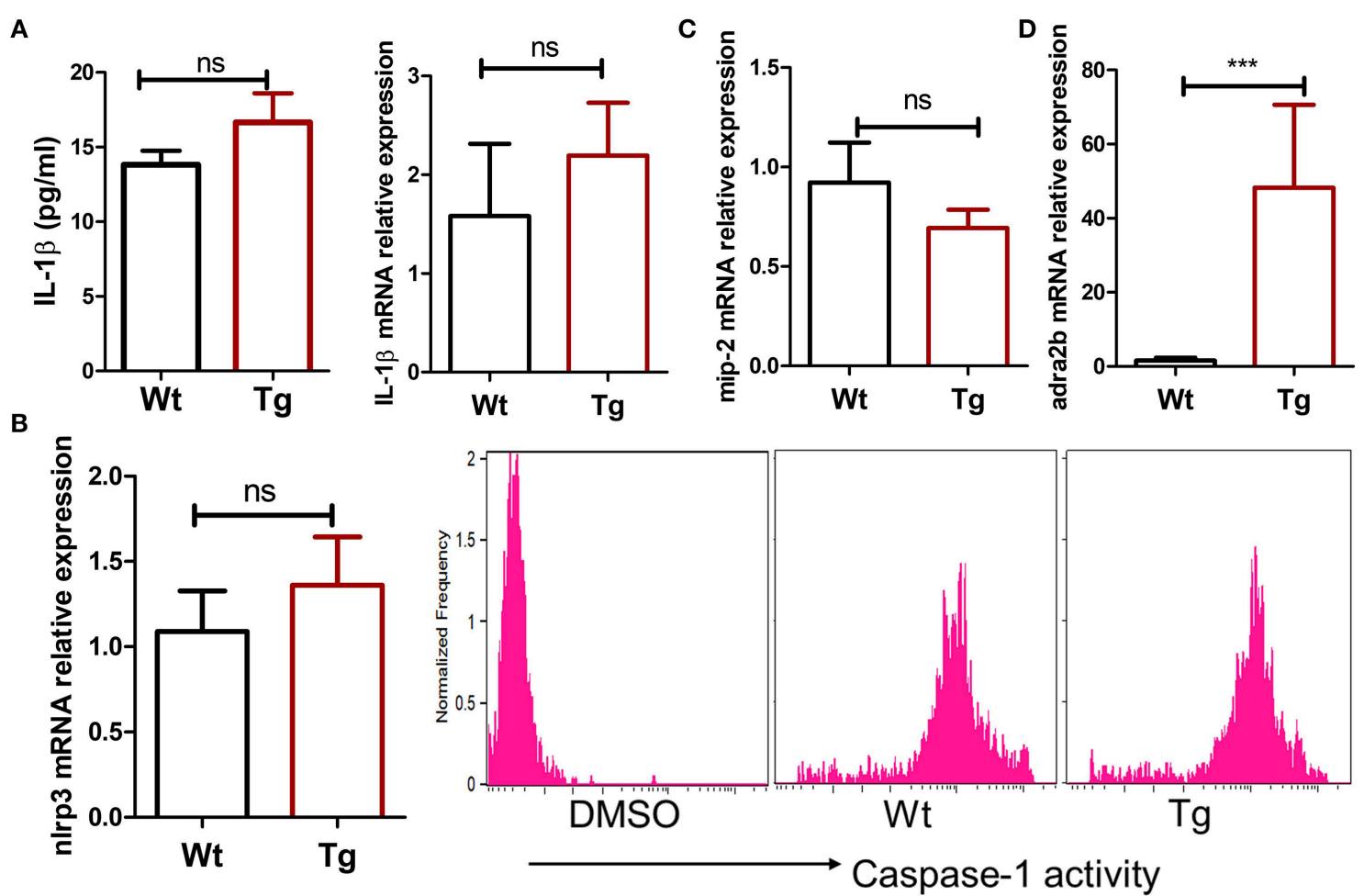

FIGURE $3 \mid \alpha_{2 B}$ AR overexpression does not affect IL-1 $\beta$ and MIP-2 production in the MSU-induced gout model. Wt and $\alpha_{2 B}$ AR-Tg mice were i.p. injected with MSU to establish the gout model. After $6 \mathrm{~h}$, the peritoneal lavage fluids and PECs were harvested. (A) The IL-1 $\beta$ levels in peritoneal lavage fluid were detected by ELISA, and mRNA expression of PECs was analyzed by real-time PCR. (B) The caspase-1 activity in peritoneal macrophage was measured by flow cytometry analysis. DMSO negative control indicates that no caspase- 1 substrates were added. (C,D) The mRNA levels of MIP-2 and $\alpha_{2 B} A R$ in PECs were measured by real-time PCR. The results shown are from one of three independent experiments $\left(n=6-8 /\right.$ group). ${ }^{\star \star \star} p<0.001$. ns, not significant.
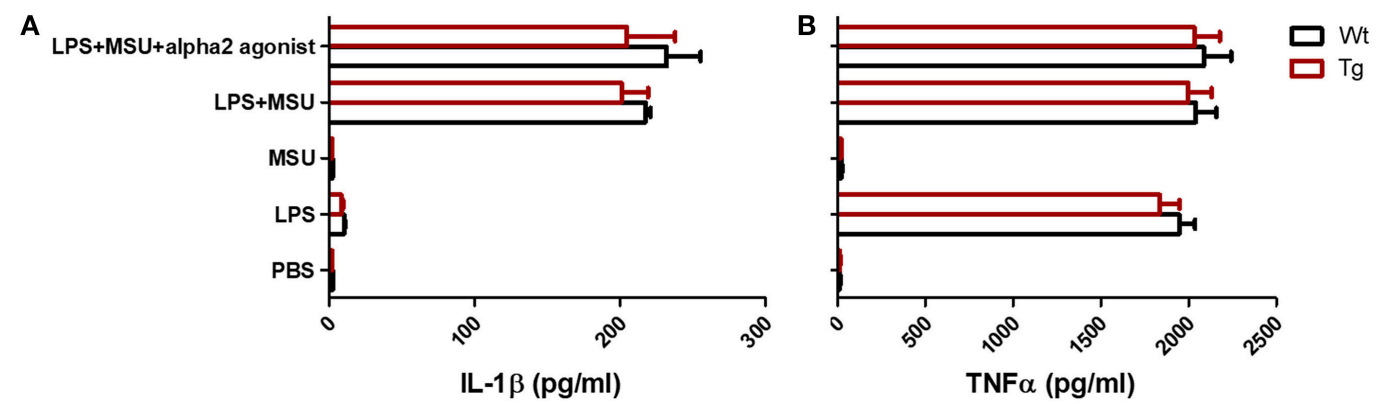

FIGURE $4 \mid \alpha_{2 B} A R$ overexpression has no effect on IL-1 $\beta$ and TNF $\alpha$ production by bone marrow derived macrophage. BMMs from $\alpha_{2 B} A R-T g$ and Wt mice were treated with LPS and/or MSU, with or without the presence of alpha2 agonist ( $n=3 /$ group). Six hours after MSU or LPS challenge, culture supernatants were collected for the detection of IL-1 $\beta$ (A) and TNF $\alpha$ (B) concentrations. Data shown is representative of 3 independent experiments. 


\section{Statistical Analysis}

Data are presented as mean \pm SEM. Group comparisons were performed using Student's $t$-test by GraphPad Prism software. $p$-values (two-tailed) below 0.05 were considered as statistically significant.

\section{RESULTS}

\section{Neutrophil Recruitment in $\alpha_{2 B} A R-T g$ Mice Is Enhanced in Gout}

Recent studies have shown a cross-communication between $\alpha_{2} \mathrm{AR}$ and innate immune response (3), while the role and mechanism of $\alpha_{2 B} A R$ subtype in the pathogenesis of gout are not clear. In this study, we first assessed the role of $\alpha_{2 B} A R$ in MSU-induced peritonitis, a mouse model of gout. At $6 \mathrm{~h}$ after MSU challenge, the total PECs were harvested for the flow cytometric analysis. Compared with the wild-type mice (Wt), $\alpha_{2 B}$ AR-Tg mice displayed a markedly elevated number of inflammatory cells in the peritoneal cavity (Figure 1A). The proportion of neutrophils, defined as $\mathrm{CD} 11 \mathrm{~b}^{+} \mathrm{Ly}_{6 \mathrm{G}}{ }^{+} \mathrm{F} 4 / 80^{-}$ cells, was significantly increased in the peritoneal cavity of $\alpha_{2 B}$ AR-Tg mice. Conversely, a decreased proportion of macrophage $\left(\mathrm{CD} 11 \mathrm{~b}^{+} \mathrm{F} 4 / 80^{+}\right)$was observed (Figures 1B,C). To further confirm the role of $\alpha_{2 B} A R$ on MSU-induced neutrophil and macrophage infiltration, the absolute number of neutrophils and macrophage in the peritoneal cavity were also calculated. The absolute number of neutrophils was considerably increased in $\alpha_{2 B} \mathrm{AR}-\mathrm{Tg}$ mice, while the absolute numbers of macrophages were similar in the $\mathrm{Wt}$ and $\alpha_{2 \mathrm{~B}} \mathrm{AR}-\mathrm{Tg}$ mice (Figure 1D). Furthermore, there was no differences in the frequency of macrophage and neutrophil in the peritoneal cavity (Figures 2A,B) and bone marrow (Figures 2C,D) between the $\alpha_{2 B} \mathrm{AR}-\mathrm{Tg}$ and Wt mice without MSU treatment. Collectively, these data demonstrated that $\alpha_{2 B} \mathrm{AR}$ overexpression promoted the development of MSU-induced inflammation.

\section{$\alpha_{2 B} A R$ Does Not Affect MSU-Stimulated IL-1 $\beta$ Production}

MSU crystals can be phagocytosed by macrophages, leading to the release of pro-inflammatory cytokines such as IL-1 $\beta, \mathrm{TNF} \alpha$, and IL- 6 . Recent studies have suggested that IL- $1 \beta$ produced by macrophage is a key inflammatory mediator in MSU crystalinduced inflammation. Massive neutrophil infiltration induced by MSU was eliminated in IL-1 receptor knockout mice (14). Since we did not observe a change in the absolute number of macrophages in $\alpha_{2 B} \mathrm{AR}-\mathrm{Tg}$ mice after MSU treatment, we next examine the ability of macrophages to produce IL-1 $\beta$. To explore whether the increased neutrophil recruitment in MSU-treated $\alpha_{2 B}$ AR-Tg mice is correlated with IL- $1 \beta$ expression, we analyzed the IL- $1 \beta$ levels of peritoneal lavage fluid from $\alpha_{2 B}$ AR-Tg mice and Wt mice treated with MSU. Unexpectedly, there was no significant difference in IL- $1 \beta$ production between $\alpha_{2 B}$ AR-Tg mice and Wt mice (Figure 3A). The maturation of active IL$1 \beta$ requires inflammasome activation (19). In consistency with

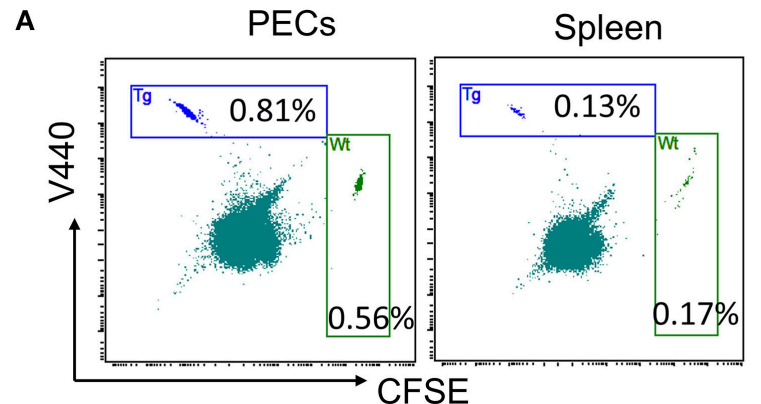

C

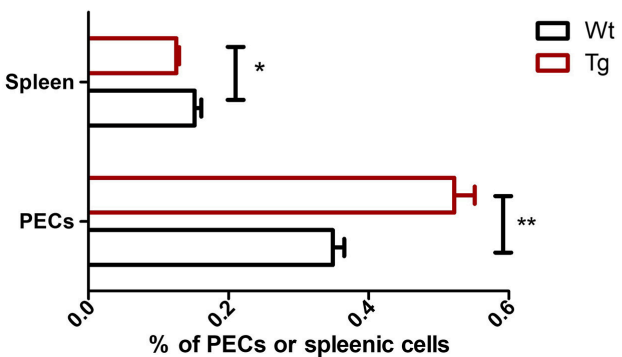

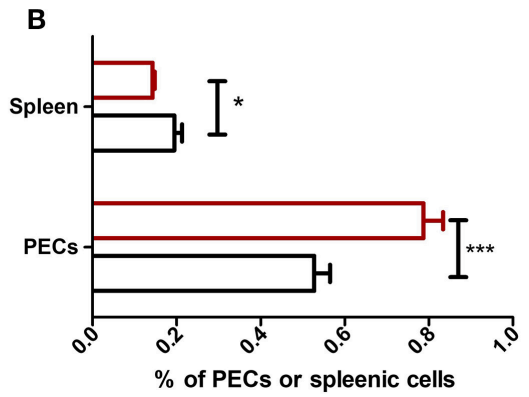

D

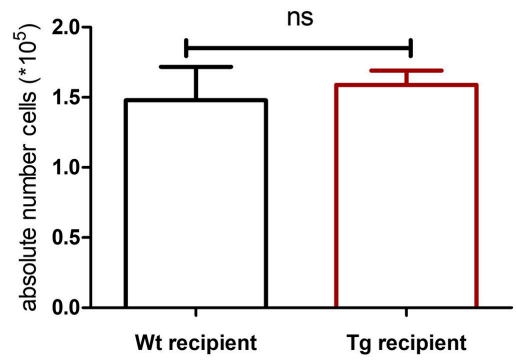

FIGURE 5 | $\alpha_{2 B} A R$ enhances neutrophil recruitment in MSU-stimulated inflammation. Bone marrow neutrophils from $\alpha_{2 B} A R-T g$ or Wt mice were stained with V440 and CFSE, respectively. Cells were then mixed at 1:1 ration, followed by i.v. injection into MSU-treated Wt mice (A,B) or $\alpha_{2 B} A R-T g$ mice (C). Six hours after MSU challenge, the PECs and spleen were harvested. (A) Representative flow cytometry analysis of neutrophil proportion in the PECs and splenic cells from MSU-treated Wt mice. (B,C) Percentages of neutrophils in PECs and spleen from MSU-treated Wt (B) and $\alpha 2 B A R-T g$ mice (C) were analyzed. (D) The peritoneal fluid was collected from Wt and $\alpha 2 B A R-T g$ recipients and the absolute number of adoptively transferred neutrophils were analyzed. Data shown are representative of 3 independent experiments ( $n=5$ /group). ${ }^{\star} p<0.05$, ${ }^{\star *} p<0.01,{ }^{\star \star *} p<0.001$. ns, not significant. 
the result of IL-1 $\beta, \alpha_{2 B} A R$ overexpression did not change the NLRP3 expression and caspase-1 activity (Figure 3B). Actually, a similar expression of MIP-2, the important chemokine of neutrophil, was observed in $\mathrm{Tg}$ and Wt mice (Figure 3C). Notably, the $\alpha_{2 B} A R$ expression of PECs was still higher in $\mathrm{Tg}$ mice than Wt mice after MSU challenge (Figure 3D). Next, we further investigated the effect of $\alpha_{2 B} A R$ overexpression on IL$1 \beta$ production by macrophages in an in vitro experiment. In accordance with the in vivo findings, there was no difference in IL- $1 \beta$ expression between $\alpha_{2 B}$ AR-Tg and Wt BMMs (Figure 4A). In addition, the production of $\mathrm{TNF} \alpha$ was also not affected by $\alpha_{2 B} A R$ overexpression (Figure 4B) Taken together, these results indicated that there is no significant difference in IL-1 $\beta$ production between $\alpha_{2 B} \mathrm{AR}-\mathrm{Tg}$ mice and Wt mice. The increased number of neutrophils in the peritoneal cavity of $\alpha_{2 B} A R-T g$ mice may be a result of enhanced migration potential of neutrophils.

\section{$\alpha_{2 B} A R$ Overexpression Enhances Neutrophil Recruitment in a Mouse Model of Gout}

To test whether the expression of $\alpha_{2 B} A R$ on neutrophils regulates their migration, neutrophils isolated from $\alpha_{2 \mathrm{~B}} \mathrm{AR}-\mathrm{Tg}$ mice and Wt mice were labeled with different fluorescent dyes, respectively, followed by adoptive transfer into MSUtreated recipients. Equal numbers of V440-labeled $\alpha_{2 B}$ AR-Tg neutrophils and CFSE-labeled Wt neutrophils were mixed and transferred into MSU-pretreated Wt mice. The PECs and splenocyes were harvested for the flow cytometric detection of V440-labeled $\alpha_{2 B}$ AR-Tg and CFSE-labeled Wt neutrophils (Figure 5A). As expected, there were more $\alpha_{2 B}$ AR-Tg neutrophils in the peritoneal cavity of the Wt recipients (Figure 5B). Moreover, $\alpha_{2 B}$ AR-Tg neutrophils had an enhanced migration into the peritoneal cavity of MSU-treated $\alpha_{2 B} A R-T g$ recipients (Figure 5C). To further examine if the expression of $\alpha_{2 B} A R$ in other tissues or cell types would affect neutrophil recruitment, we compared the migration of neutrophils into the peritoneal cavity of Wt vs. $\alpha_{2 B} A R-T g$ mice. We found the absolute number of neutrophils migrated to the peritoneal cavity of $\alpha_{2 B} \mathrm{AR}-\mathrm{Tg}$ recipients was similar to that migrated into the peritoneal cavity of $\mathrm{Wt}$ recipients (Figure 5D), suggesting the overexpression of $\alpha_{2 B} A R$ in other tissue or cell types did not affect the migratory ability of neutrophils. These data indicated that $\alpha_{2 B} A R$ overexpression on neutrophils promotes its migratory ability.
A

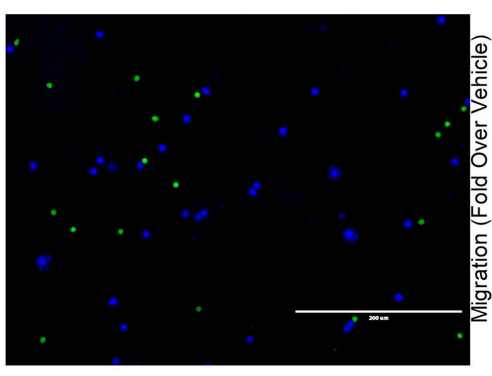

C
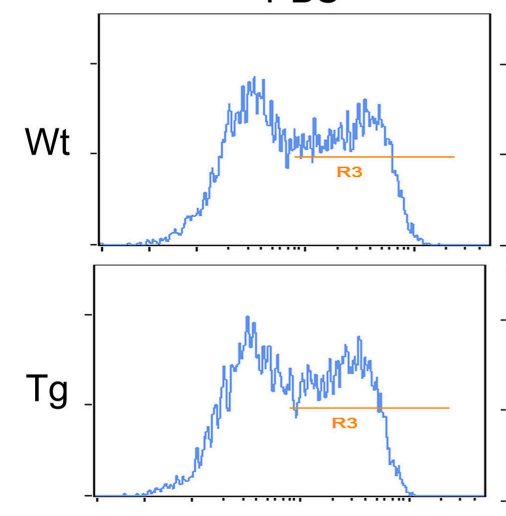
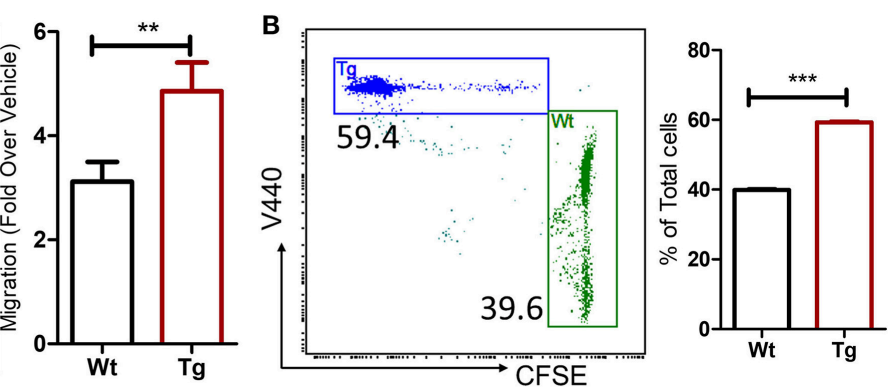

MIP-2

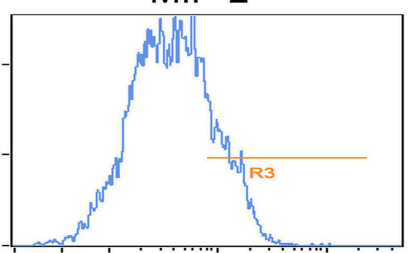

MIP-2+agonist
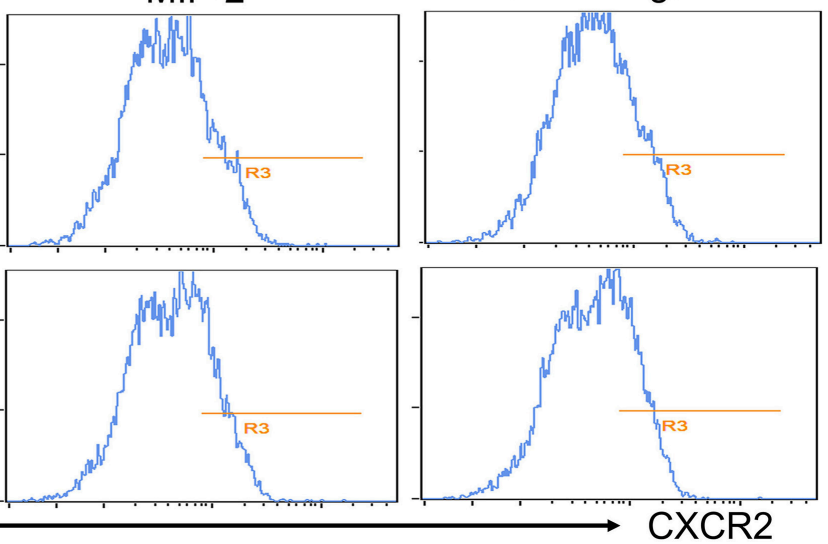

FIGURE 6 | $\alpha_{2 B} A R$ overexpression enhances in vitro neutrophils migration. Neutrophils were isolated from $\alpha 2 B A R-T g$ and Wt bone marrows and stained with V440 (blue) and CFSE (green), respectively. Labeled cells were mixed at 1:1 ration and used for the Transwell ${ }^{\circledR}$ migration assay in response to MIP2. (A) Upper panel, Representative fluorescent microscopic image showing $\alpha 2 B A R-T g$ (Blue) and Wt (Green) neutrophils migrating through the membrane. Lower panel, quantitative analysis showing the migration of $\alpha 2 B A R-T g$ and Wt neutrophils. (B) Representative dot plot (upper panel) and quantitative analysis (lower panel) showing the percentages of V440-labeled cells ( $\alpha 2 \mathrm{BAR}-\mathrm{Tg}$ ) and CFSE-labeled cells $(\mathrm{Wt})$ in the bottom well of the Transwell ${ }^{\circledR}$ plate. (C) The Cxcr2 expressions on bone marrows neutrophil with or without treatment of MIP-2 or MIP-2 plus $\alpha 2 \mathrm{BAR}$ agonist clonidine hydrochloride were analyzed by flow cytometry. Data shown are representative of 3 independent experiments. ${ }^{\star *} p<0.01,{ }^{* \star *} p<0.001$. 


\section{$\alpha_{2 B} A R$ Overexpression Enhances the Migratory Capacity of Neutrophil in vitro}

Because lots of factors can affect the neutrophil migration during the development of inflammation in vivo, the migratory capacity of $\alpha_{2 B} \mathrm{AR}-\mathrm{Tg}$ neutrophils was further investigated in vitro to exclude the microenvironmental factors. CXCR2 has a central role in the recruitment of neutrophils. We next examined the migration of the neutrophils toward CXCR2 ligand MIP-2. In consistency with the in vivo result, $\alpha_{2 B} \mathrm{AR}$ overexpression on neutrophils increased their chemotaxis toward MIP-2 in the in vitro Transwell ${ }^{\circledR}$ assay (Figures 6A,B). We next examined the expression level of CXCR2 on neutrophils. No significant difference in CXCR2 expressions on bone marrow neutrophils was observed between $\alpha_{2 B} \mathrm{AR}-\mathrm{Tg}$ and Wt mice (Figure 6C). It has been shown that the binding with MIP-2 induces CXCR2 internalization. In consistency, we here also observed a decreased expression on neutrophil surface after MIP-2 treatment in both $\mathrm{Wt}$ and $\mathrm{Tg}$ mice. There was also no significant difference in cell surface expression of CXCR2 after MIP-2 stimulation, suggesting that $\alpha_{2 B} \mathrm{AR}$ does not affect CXCR2 internalization (Figure 6C). In addition, $\alpha_{2 B} A R$ activation by agonist clonidine hydrochloride did not affect the expression of CXCR2, further suggesting that the enhanced neutrophil migration was independent of CXCR2 expression (Figure 6C). These data strongly suggested that the increased migratory ability of $\alpha_{2 B} \mathrm{AR}-\mathrm{Tg}$ neutrophils was independent of CXCR2.

\section{DISCUSSION}

In this study, we showed that MSU-induced inflammation was enhanced in $\alpha_{2 B} A R-T g$ mice, characterized by increased neutrophil infiltration. $\alpha_{2 B} A R$ overexpression did not affect the IL- $1 \beta$ production by macrophage, while the migratory ability of neutrophils was significantly enhanced in $\alpha_{2 B}$ ARoverexpressing neutrophils.

Increasing evidence show that adrenergic receptors have a close relationship with immune system. All the three families of AR have already been reported to play a role in regulating innate immune responses and immune-related diseases $(2,20)$. For instance, $\beta$ ARs are highly and widely expressed in immune cells. They regulate a number of functions, including lymphocytes homing and maturation $(8,21,22)$. Previous studies have also shown a negative influence of norepinephrine on NK cell activity via $\beta_{2}$ ARs (23). In our study, we found for the first time that there was a significantly increased number of inflammatory cells in peritoneal cavity of $\alpha_{2 B}$ AR-Tg mice upon MSU treatment. IL-1 $\beta$ is considered as a critical pro-inflammatory cytokine in the gout, with a wide range of systemic and local effects. In humans, the production of IL- $1 \beta$ by resident macrophages is stimulated by MSU crystal and plays as a crucial role in the pathogenesis of gout (14). Moreover, IL-1 $\beta$ has the ability to induce neutrophilia and neutrophil migration (24). It has been reported that exposure of macrophages to lipopolysaccharide led to a release of catecholamines and there is an autocrine/paracrine self-regulatory mechanism in catecholamine production by macrophages during inflammation (25). Blockade of $\alpha_{2} \mathrm{AR}$ or catecholamine-generating enzymes greatly suppressed LPSinduced inflammation (25). Catecholamines has been shown to be able to promote NF- $\mathrm{\kappa B}$ signaling in macrophages via activating $\alpha_{2} A R$, leading to an amplification of the acute inflammatory response (26). However, we did not observe an increased IL- $1 \beta$ production and caspase- 1 activity in the $\alpha_{2 B}$ AR-overexpressing macrophages in our study. These data demonstrated that increased cytokines production induced by catecholamines may be independent of $\alpha_{2 B}$-AR stimulation.

As an early event in immune response against invading pathogens, accumulation of neutrophils was also an early and critical step in sterile inflammation, such as gout and ischemia/reperfusion $(27,28)$. Inflammatory stimuli induce neutrophil mobilization from bone marrow, resulting in neutrophilia and neutrophil infiltration into the affected local area (29). In consistency with this, the number of neutrophil is significantly increased in the patients with acute gout. Both vagal parasympathetic and sympathetic signals have been shown to regulate inflammation through activating cholinergic receptors on macrophage and neutrophils $(3,30-32)$. It has been demonstrated that norepinephrine can induce immediate neutrophil mobilization through acting on $\alpha$-AR (33), and leukocyte-expressing $\beta_{2}$-AR has been found to be essential for survival after acute myocardial injury (34). Propranolol, a $\beta_{1^{-}}$$\mathrm{AR} / \beta_{2}-\mathrm{AR}$ inhibitor, is able to reduce the numbers of circulating neutrophils, suppress neutrophil infiltration into colonic tissues, and attenuate the colonic tissue damage in IBD (35). Here, the increase of the inflammatory cell number in MSU-treated $\alpha_{2 B}$ AR$\mathrm{Tg}$ mice was identified to a result of enhanced migratory ability of neutrophils as the absolute number of macrophage did not change. Furthermore, the number of neutrophils in bone marrow was similar between treatment naive $\alpha_{2 \mathrm{~B}} \mathrm{AR}-\mathrm{Tg}$ and WT mice, excluding the possibility that $\alpha_{2 \mathrm{~B}} \mathrm{AR}$ may regulate neutrophil differentiation. The regulation of neutrophil function by SNS may vary in different diseases. Our findings indicate that $\alpha_{2 B} A R$ overexpression might play a critical role in the development of gout through promoting neutrophil recruitment into the inflammatory sites.

The migration process of neutrophil includes the following steps. The initial step of neutrophils is movement from the central stream to the periphery of a vessel, which allows the cell surface molecular interaction between neutrophil and vessel endothelial cell and then neutrophil rolling on the endothelial cells. After that, a chemoattractant gradient produced by local inflammation elicits the transmigration process of neutrophils (27). In our study, no difference in IL-1 $\beta$ production was observed between $\mathrm{Wt}$ and $\alpha_{2 \mathrm{~B}} \mathrm{AR}-\mathrm{Tg}$ mice. In addition, mixed $\mathrm{Wt}$ and $\alpha_{2 \mathrm{~B}} \mathrm{AR}-$ $\mathrm{Tg}$ neutrophils displayed different migratory ability in the same environment, regardless in Wt or $\alpha_{2 B} A R-T g$ recipients. Furthermore, Wt and $\alpha_{2 \mathrm{~B}} \mathrm{AR}-\mathrm{Tg}$ neutrophils had an enhanced migration toward MIP2, while the CXCR2 receptor expression was similar to Wt neutrophils. These data suggest that the enhanced migratory ability in $\alpha_{2 B}$ AR-overexpressing neutrophils is independent of chemokine and chemokine receptors. Further investigations are required to understand the exact mechanisms by which $\alpha_{2 B} A R$ signals regulate the mobility of neutrophils. 
Taken together, we demonstrated that $\alpha_{2 B} A R$ is involve in the progress of MSU-induced inflammation and $\alpha_{2 B} \mathrm{AR}$ might be a promising therapeutic target for gout.

\section{DATA AVAILABILITY}

All datasets generated for this study are included in the manuscript and/or the supplementary files.

\section{AUTHOR CONTRIBUTIONS}

$\mathrm{LD}, \mathrm{XR}$, and JZ conceived the project, designed and carried out some experiments, analyzed all data, and wrote the paper. JC were responsible for design and performance of experiments, and contributed to analyzing data and writing the paper. YW, $\mathrm{MR}$, and YT helped in performing all the experiments, analyzing all data, generating figures of the data. All the authors read,

\section{REFERENCES}

1. Calcagni E, Elenkov I. Stress system activity, innate and T helper cytokines, and susceptibility to immune-related diseases. Ann N Y Acad Sci. (2006) 1069:62-76. doi: 10.1196/annals.1351.006

2. Bellinger DL, Millar BA, Perez S, Carter J, Wood C, ThyagaRajan S, et al. Sympathetic modulation of immunity: relevance to disease. Cell Immunol. (2008) 252:27-56. doi: 10.1016/j.cellimm.2007.09.005

3. Padro CJ, Sanders VM. Neuroendocrine regulation of inflammation. Semin Immunol. (2014) 26:357-68. doi: 10.1016/j.smim.2014.01.003

4. Verburg-van Kemenade BM, Van der Aa LM, Chadzinska M. Neuroendocrine-immune interaction: regulation of inflammation via G-protein coupled receptors. Gen Comp Endocrinol. (2013) 188:94-101. doi: 10.1016/j.ygcen.2012.11.010

5. Henry JP. Biological basis of the stress response. Integr Physiol Behav Sci. (1992) 27:66-83. doi: 10.1007/BF02691093

6. Johnson JD, Campisi J, Sharkey CM, Kennedy SL, Nickerson M, Greenwood $\mathrm{BN}$, et al. Catecholamines mediate stress-induced increases in peripheral and central inflammatory cytokines. Neuroscience. (2005) 135:1295-307. doi: 10.1016/j.neuroscience.2005.06.090

7. Elenkov IJ, Kvetnansky R, Hashiramoto A, Bakalov VK, Link AA, Zachman $\mathrm{K}$, et al. Low- versus high-baseline epinephrine output shapes opposite innate cytokine profiles: presence of Lewis- and Fischer-like neurohormonal immune phenotypes in humans? J Immunol. (2008) 181:1737-45. doi: 10.4049/jimmunol.181.3.1737

8. Lubahn CL, Lorton D, Schaller JA, Sweeney SJ, Bellinger DL. Targeting alphaand beta-adrenergic receptors differentially shifts Th1, Th2, and inflammatory cytokine profiles in immune organs to attenuate adjuvant arthritis. Front Immunol. (2014) 5:346. doi: 10.3389/fimmu.2014.00346

9. Brosnan CF, Goldmuntz EA, Cammer W, Factor SM, Bloom BR, Norton WT. Prazosin, an alpha 1-adrenergic receptor antagonist, suppresses experimental autoimmune encephalomyelitis in the Lewis rat. Proc Natl Acad Sci USA. (1985) 82:5915-9. doi: 10.1073/pnas.82.17.5915

10. Capellino S, Straub RH. Neuroendocrine immune pathways in chronic arthritis. Best Pract Res Clin Rheumatol. (2008) 22:285-97. doi: 10.1016/j.berh.2008.01.010

11. Straub RH, Grum F, Strauch U, Capellino S, Bataille F, Bleich A, et al. Antiinflammatory role of sympathetic nerves in chronic intestinal inflammation. Gut. (2008) 57:911-21. doi: 10.1136/gut.2007.125401

12. Manolis AJ, Poulimenos LE, Kallistratos MS, Gavras I, Gavras H. Sympathetic overactivity in hypertension and cardiovascular disease. Curr Vasc Pharmacol. (2014) 12:4-15. doi: 10.2174/15701611 113119990140 critically revised, and agreed to be accountable for the content of manuscript.

\section{FUNDING}

This work was supported by grants from National Natural Science Foundation of China (81670431 and 31870906, 81671544 and 81871286), National Institutes of Health (K01DK105108, R03DK119680, and K99ES026241), American Heart Association (17GRNT33670485), American Diabetes Association (1-19-JDF117), and American Association of Immunologists (CIIF-8745).

\section{ACKNOWLEDGMENTS}

We are extremely grateful to Yan $\mathrm{He}$ who performed flow cytometry analysis in this study. JC gratefully acknowledged financial support from China Scholarship Council.

13. Snitker S, Macdonald I, Ravussin E, Astrup A. The sympathetic nervous system and obesity: role in aetiology and treatment. Obes Rev. (2000) 1:5-15. doi: 10.1046/j.1467-789x.2000.00001.x

14. So A, Dumusc A, Nasi S. The role of IL-1 in gout: from bench to bedside. Rheumatology. (2018) 57(suppl_1):i12-i9. doi: 10.1093/rheumatology/kex449

15. Newberry SJ, FitzGerald JD, Motala A, Booth M, Maglione MA, Han D, et al. Diagnosis of gout: a systematic review in support of an american college of physicians clinical practice guideline. Ann Intern Med. (2017) 166:27-36. doi: 10.7326/M16-0462

16. Yanagawa $Y$, Matsumoto $M$, Togashi H. Enhanced dendritic cell antigen uptake via alpha2 adrenoceptor-mediated PI3K activation following brief exposure to noradrenaline. J Immunol. (2010) 185:5762-8. doi: 10.4049/jimmunol.1001899

17. Tanabe K, Matsushima-Nishiwaki R, Kozawa O, Iida H. Dexmedetomidine suppresses interleukin-1beta-induced interleukin-6 synthesis in rat glial cells. Int J Mol Med. (2014) 34:1032-8. doi: 10.3892/ijmm.2014.1863

18. Zhong J, Rao X, Braunstein Z, Taylor A, Narula V, Hazey J, et al. T-cell costimulation protects obesity-induced adipose inflammation and insulin resistance. Diabetes. (2014) 63:1289-302. doi: 10.2337/db13-1094

19. Martinon F, Petrilli V, Mayor A, Tardivel A, Tschopp J. Gout-associated uric acid crystals activate the NALP3 inflammasome. Nature. (2006) 440:237-41. doi: $10.1038 /$ nature 04516

20. Dantzer R. Neuroimmune interactions: from the brain to the immune system and vice versa. Physiol Rev. (2018) 98:477-504. doi: 10.1152/physrev.00039.2016

21. Nakai A, Hayano Y, Furuta F, Noda M, Suzuki K. Control of lymphocyte egress from lymph nodes through beta2-adrenergic receptors. J Exp Med. (2014) 211:2583-98. doi: 10.1084/jem.20141132

22. Beiermeister KA, Keck BM, Sifri ZC, ElHassan IO, Hannoush EJ, Alzate WD, et al. Hematopoietic progenitor cell mobilization is mediated through beta-2 and beta-3 receptors after injury. J Trauma. (2010) 69:338-43. doi: 10.1097/TA.0b013e3181e5d35e

23. Takamoto T, Hori Y, Koga Y, Toshima H, Hara A, Yokoyama MM. Norepinephrine inhibits human natural killer cell activity in vitro. Int J Neurosci. (1991) 58:127-31. doi: 10.3109/00207459108987189

24. Dinarello CA. Immunological and inflammatory functions of the interleukin-1 family. Annu Rev Immunol. (2009) 27:519-50. doi: 10.1146/annurev.immunol.021908.132612

25. Flierl MA, Rittirsch D, Nadeau BA, Chen AJ, Sarma JV, Zetoune FS, et al. Phagocyte-derived catecholamines enhance acute inflammatory injury. Nature. (2007) 449:721-5. doi: 10.1038/nature06185

26. Flierl MA, Rittirsch D, Nadeau BA, Sarma JV, Day DE, Lentsch AB, et al. Upregulation of phagocyte-derived catecholamines augments 
the acute inflammatory response. PLoS ONE. (2009) 4:e4414. doi: 10.1371/journal.pone.0004414

27. Nathan C. Neutrophils and immunity: challenges and opportunities. Nat Rev Immunol. (2006) 6:173-82. doi: 10.1038/nri1785

28. Amulic B, Cazalet C, Hayes GL, Metzler KD, Zychlinsky A. Neutrophil function: from mechanisms to disease. Annu Rev Immunol. (2012) 30:459-89. doi: 10.1146/annurev-immunol-020711074942

29. Weiss SJ. Tissue destruction by neutrophils. N Engl J Med. (1989) 320:365-76. doi: 10.1056/NEJM198902093200606

30. Sellegounder D, Yuan CH, Wibisono P, Liu Y, Sun J. Octopaminergic signaling mediates neural regulation of innate immunity in Caenorhabditis elegans. MBio. (2018) 9:e01645-18. doi: 10.1128/mBio. 01645-18

31. Aballay A. Neural regulation of immunity: role of NPR-1 in pathogen avoidance and regulation of innate immunity. Cell Cycle. (2009) 8:966-9. doi: $10.4161 /$ cc.8.7.8074

32. Sternberg EM. Neural regulation of innate immunity: a coordinated nonspecific host response to pathogens. Nat Rev Immunol. (2006) 6:318-28. doi: 10.1038/nri1810

33. Beis D, von Kanel R, Heimgartner N, Zuccarella-Hackl C, Burkle A, Ehlert $\mathrm{U}$, et al. The role of norepinephrine and alpha-adrenergic receptors in acute stress-induced changes in granulocytes and monocytes. Psychosom Med. (2018) 80:649-58. doi: 10.1097/PSY.0000000000000620

34. Grisanti LA, Gumpert AM, Traynham CJ, Gorsky JE, Repas AA, Gao E, et al. Leukocyte-Expressed beta2-adrenergic receptors are essential for survival after acute myocardial injury. Circulation. (2016) 134:153-67. doi: 10.1161/CIRCULATIONAHA.116.022304

35. Deng Q, Chen H, Liu Y, Xiao F, Guo L, Liu D, et al. Psychological stress promotes neutrophil infiltration in colon tissue through adrenergic signaling in DSS-induced colitis model. Brain Behav Immun. (2016) 57:24354. doi: 10.1016/j.bbi.2016.04.017

Conflict of Interest Statement: The authors declare that the research was conducted in the absence of any commercial or financial relationships that could be construed as a potential conflict of interest.

Copyright (C) 2019 Duan, Chen, Razavi, Wei, Tao, Rao and Zhong. This is an openaccess article distributed under the terms of the Creative Commons Attribution License (CC BY). The use, distribution or reproduction in other forums is permitted, provided the original author(s) and the copyright owner(s) are credited and that the original publication in this journal is cited, in accordance with accepted academic practice. No use, distribution or reproduction is permitted which does not comply with these terms. 